\title{
Metastatic Colorectal Carcinoma after Second Progression and the Role of Trifluridine-Tipiracil (TAS-102) in Switzerland
}

\author{
Alexander Siebenhüner $^{\mathrm{a}}$ Sara De Dosso $^{\mathrm{b}} \quad$ Alexander Meisel $^{\mathrm{c}}$ \\ Anna Dorothea Wagner ${ }^{d}$ Markus Borner ${ }^{\mathrm{e}}$ \\ ${ }^{a}$ Clinic for Medical Oncology and Hematology, Universitätsspital Zürich and University of Zurich, Zurich, \\ Switzerland; 'b Istituto Oncologico della Svizzera Italiana, Bellinzona, Switzerland; ' ${ }^{C}$ Hematology and Oncology, \\ Clinic for Internal Medicine, Stadtspital Waid, Zurich, Switzerland; 'Département d'oncologie, CHUV, Lausanne, \\ Switzerland; ${ }^{e}$ ONCOCARE at the Engeriedspital, Bern, Switzerland
}

\section{Keywords}

Refractory colorectal cancer · Lonsurf · Trifluridine-tipiracil · Tas-102 $\cdot$ Rechallenge $\cdot$ Regorafenib $\cdot$ Second progression

\begin{abstract}
Background: Metastatic colorectal carcinoma ( $\mathrm{mCRC}$ ) is one of the most prevalent types of cancer worldwide. After tumor progression with first- and second-line treatment, trifluridine (FTD) and tipiracil (TPI) has been shown to be a treatment option. Summary: Data from a pivotal phase 3 trial (RECOURSE) and an ongoing phase $3 \mathrm{~b}$ trial (PRECONNECT) have shown that, in $\mathrm{mCRC}$ patients who experienced disease progression after 2 lines of standard therapy, treatment with FTD/TPI is safe and efficacious. Other third-line options include regorafenib, rechallenge with previous treatment lines or personalized approaches based on comprehensive molecular profiling. Randomized trials or sequential studies aiming for the right treatment sequence or predefined subtypes for FTD/TPI or regorafenib as well for rechallenge are missing. However, FTD/TPI as well as regorafenib are recommended by the current ESMO, German S3, and National Comprehensive Cancer Network (NCCN) guidelines in the same situation, thus offering physicians a number of alternatives for the treatment of $\mathrm{mCRC}$ patients after the second progression. Key Message: This narrative review summariz-
\end{abstract}

karger@karger.com www.karger.com/ort

Karger ${ }^{\prime \prime}=$ (c) 2020 The Author(s) Karger This article is licensed under the Creative Commons AttributionUsage and distribution for commercial purposes as well as any Published by S. Karger AG, Basel Open access NonCommercial-NoDerivatives 4.0 International License (CC BYNC-ND) (http://www.karger.com/Services/OpenAccessLicense). es published data and their impact for FTD/TPI as well for regorafenib and rechallenge chemotherapy in clinical practice settings of refractory situations of colorectal cancer.

(c) 2020 The Author(s)

Published by S. Karger AG, Basel

\section{Introduction}

Colorectal cancer (CRC) is the third most common type of cancer worldwide, accounting for $10.6 \%$ of all cancer cases [1]. In Switzerland, about 4,000 new patients are diagnosed with CRC every year, which is $11 \%$ of all cancer diagnoses [2]. The majority of diagnoses are staged in a metastatic setting. At that stage most tumors are inoperable and systemic treatment is recommended. Generally, metastatic colorectal carcinoma (mCRC) patients receive multiple lines of therapy during their history of disease [3]. However, in Switzerland, management of mCRC is challenging, as no Switzerland-specific guidelines for mCRC treatment have been established until now. Thus, Swiss physicians use international recommendations for mCRC as listed in ESMO, German S3, and National Comprehensive Cancer Network (NCCN) guidelines, which vary in nuances from each other [3-5]. Basically, the establishment of treatment recommendations with ongoing 
updates presented in these guidelines has led to an overall improvement in the clinical outcome of mCRC patients [6]. Different combinations of chemotherapies with or without biologicals or monotherapy regimens or even immunotherapies based on the molecular profile as well the location of the tumor drives the choice of first- and second-line treatment regimens for mCRC [3-5]. These strategies have resulted in improvement of overall survival (OS) of mCRC patients, i.e., from 19 months to over 3 years in the last decade [7]. As mCRC patients tend to live longer with the tumor, a larger group of patients should be assessed for third or refractory lines of treatment. In cases of refractory mCRC that has not responded favorably to first- and second-line treatments, an oral combination drug, i.e., trifluridine-tipiracil (FTD/TPI, Lonsurf ${ }^{\circledR}$ ), or an oral multikinase inhibitor, i.e., regorafenib, as well as rechallenge of chemotherapy with or without a formerly used antibody regimen are valid options presented in ESMO and NCCN recommendations $[3,5]$. The recommendation level in the guidelines of the NCCN and ESMO ranks these options equally, whereas $\mathrm{S} 3$ has reassessed the ranking of regorafenib with a weaker recommendation compared to other options such as FTD/TPI or rechallenge. This mainly affects mCRC patients in Germany as regorafenib is not directly available in that country [3-5].

The other oral drug for refractory $\mathrm{mCRC}$ is FTD/TPI. It is a combination of: (a) the thymidine-based nucleoside analog FTD, which has been reported to act through inhibition of thymidylate synthase and incorporation into the DNA, ultimately leading to DNA damage and cell death, and (b) the TPase inhibitor TPI, which prevents the rapid degradation of FTD, thus elongating its short half-life. As a result, FTD/TPI remains in the body longer and can therefore be administered at a lower dose. Since the mode of action of FTD/TPI is significantly different from that of fluorouracil (5-FU) (Fig. 1), it has been shown to have considerable efficacy in 5-FU-refractory patients [8]. In June 2015, Servier and Taiho Pharmaceutical obtained an exclusive license for FTD/TPI under the name Lonsurf ${ }^{\circledR}$ [9] for Japan, the USA, the EU, and Switzerland. It has been licensed for adult $\mathrm{mCRC}$ patients who have already been treated with available first- and second-line therapies such as fluoropyrimidine (5-FU/ capecitabine)-, oxaliplatin- and irinotecan-based chemotherapy and anti-VEGF therapy and for RAS wild-type mCRC patients treated with anti-EGFR therapy [3-5].

\section{Studies Favoring FTD/TPI Treatment for mCRC Patients}

A preliminary analysis of the data from the ongoing multicenter phase $3 \mathrm{~b}$ study PRECONNECT, designed to evaluate the safety and efficacy of FTD/TPI in real-life conditions, confirms the advantages of FTD/TPI as a favorable treatment for refractory mCRC [10]. A preliminary analysis of data from 462 patients showed that patients on FTD/TPI had a median progression-free survival (PFS) of 2.8 months, disease control was achieved in $37 \%$ of the patients, and their performance status deteriorated to ECOG performance status $\geq 2$ after a median of only 8.7 months [10]. The safety profile of the therapy was acceptable and consistent with that found in the RECOURSE study - a pivotal phase 3 trial comparing the efficacy of FTD/TPI to that od placebo for the treatment of refractory CRC and $\mathrm{mCRC}$ in heavily pretreated patients (ECOG performance status 0-1) [11,12]. The RECOURSE study demonstrated that FTD/TPI treatment successfully prolonged the median OS by about 2 months (Fig. 2) and PFS (2 vs. 1.7 months) with an acceptable toxicity profile $[11,12]$. The most frequently observed side effects with FTD/TPI were neutropenia (32.4\%), asthenia (24.3\%), anemia (21.6\%), and diarrhea (18.9\%) [12]. A retrospective analysis showed that neutropenia could be a good surrogate marker for adequate dose exposure [13]. Furthermore, all of the subgroups in the patient data set, including the geographical subsets, the KRAS mutation-based subsets, etc., benefited from FTD/TPI treatment $[11,12]$. A number of phase $1 / 2$ studies have also shown that the combination of FTD/TPI and bevacizumab can lead to a positive clinical outcome. A recent study demonstrated that the addition of bevacizumab to an FTD/TPI treatment regimen had a tolerable toxicity profile and led to a prolonged median PFS (from 2.6 to 5.9 months, 95\% CI $0.28-0.92 ; p<0.03$ ) and median OS (from 7.3 to 10.3 months, 95\% CI 0.18-0.99; $p<0.05$ ) [14]. This study was inspired by a phase $1 / 2$ multicenter Japanese study (CTASK FORCE), which also showed similar results [15]. Neutropenia (72\%) and leucopenia (44\%) were the most common adverse effects observed in this study [15]. Furthermore, a phase 2 trial, i.e., RAMTAS, is currently assessing the efficacy of the combination of an anti-VEGFR antibody, ramucirumab, and FTD/TPI [16].

Several compassionate use programs of FTD/TPI across Europe, the USA, and Asia have been conducted and in summary they underline the efficacy and safety of FTD/TPI in a real-world population with refractory mCRC [17-21].

\section{Rechallenge Therapy: an Alternative after a Second Progression}

Rechallenge therapy, i.e., administration of a previously given regimen of chemotherapy with or without the same antibodies (e.g., bevacizumab or anti-EGFR related to a molecular tumor profile), may be an option for selected patients [22]. 
Fig. 1. Mechanism of action of FTD/TPI. FTD/TPI shows a different mechanism of action compared to 5-FU-based fluoropyrimidines. Adapted from Lenz et al. [8].

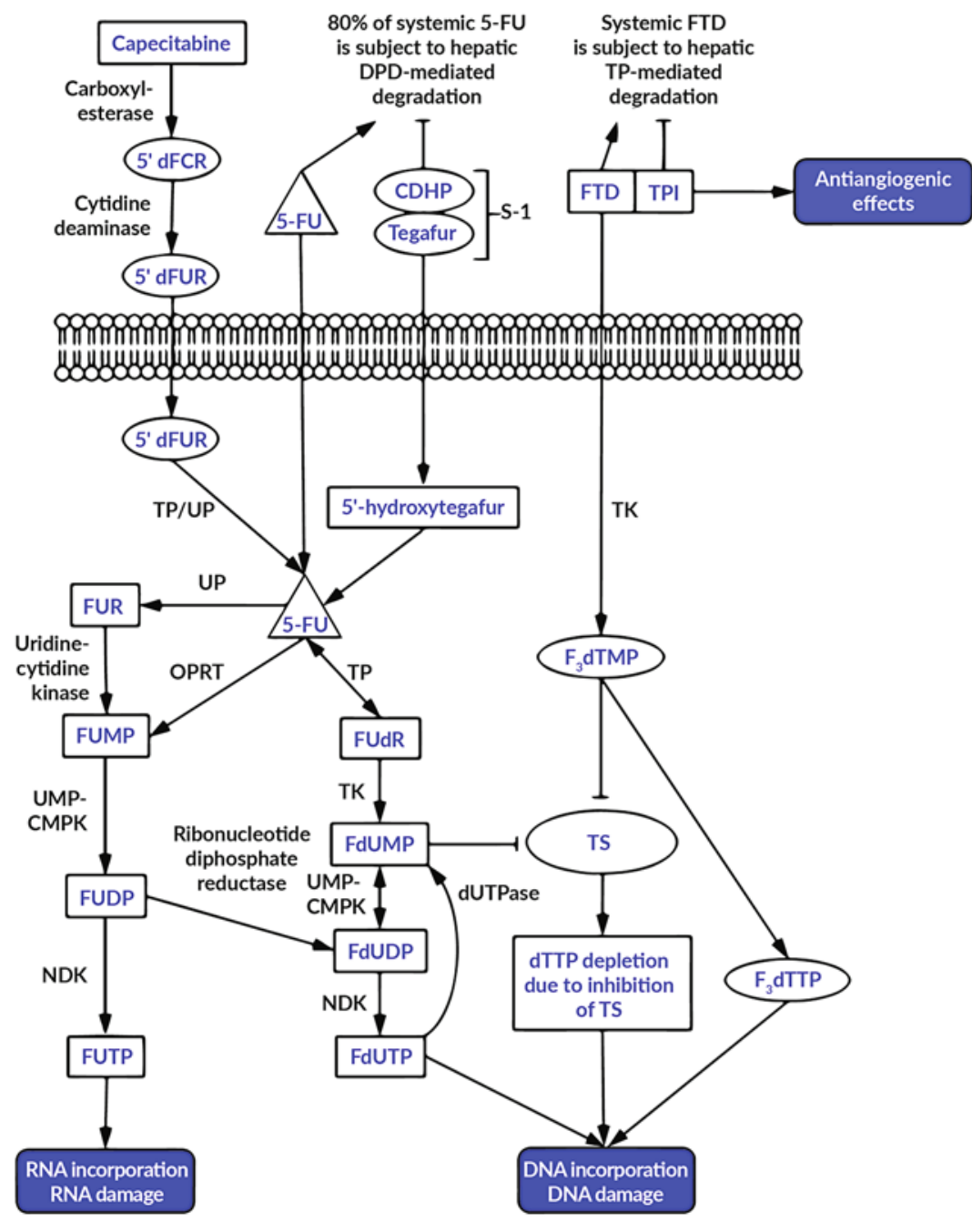

A possible explanation for the efficacy of such a rechallenge strategy is that $\mathrm{mCRC}$ comprises heterogeneous tumor clones. Therefore, the first- and second-line treatment do not entirely address this heterogeneity. Even clones that were suppressed do not completely disappear during the initial treatment and can initiate disease progression at a later point. Since the number of cytostatic agents available for mCRC therapy is limited, resumption of treatment with the same chemotherapeutic agents can represent a treatment continuum.

This rechallenge concept especially attracts the situation if more than 6 months have elapsed since the last use of this specific regimen. In a prospective study with mCRC patients who presented, at the first stage, a response to aEGFR but developed resistance over time were rechallenged with the aEGFR antibody cetuximab plus irinotecan in the refractory setting [23]. Several other studies like OPTIMOX 2, COIN, or NORDIC IV underline the concept of rechallenge even using a different chemotherapy backbone in these patients [24]. Interestingly, these highly selected patient benefit from this third-line strategy. A precondition is that no RAS or BRAF mutation was detected by liquid biopsy prior to rechallenge therapy [25].

The fact that a median OS of up to 18 months was achieved in the REVERSE study [26] - which is longer than that observed after treatment with licensed second-line treatments such as aflibercept-FOLFIRI [27] strongly supports the idea that this is a highly selective patient group. A benefit of rechallenge with an anti-EGFR antibody was demonstrated by biomarkers and evidence of the EGFR wild-type clone [25]. Moreover, excellent re- 
A Overall Survival

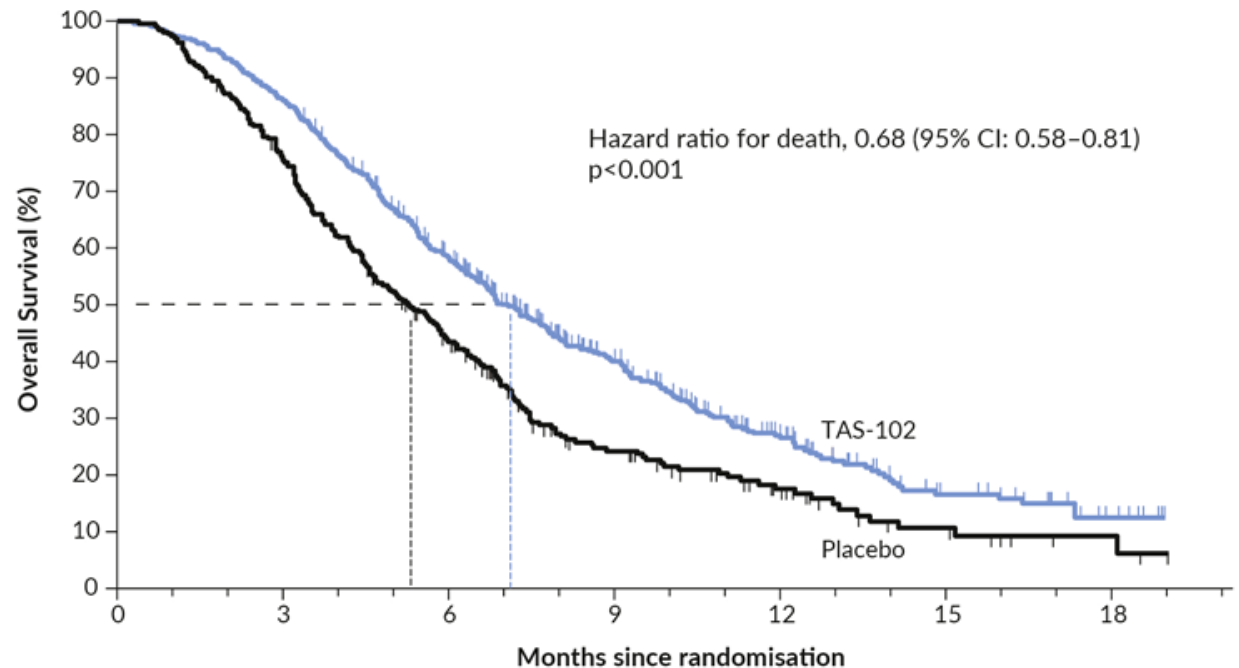

No. at Risk

$\begin{array}{lrrrrrrr}\text { TAS-102 } & 534 & 459 & 294 & 137 & 64 & 23 & 7 \\ \text { Placebo } & 266 & 198 & 107 & 47 & 24 & 9 & 3\end{array}$

\section{B Overall Survival}

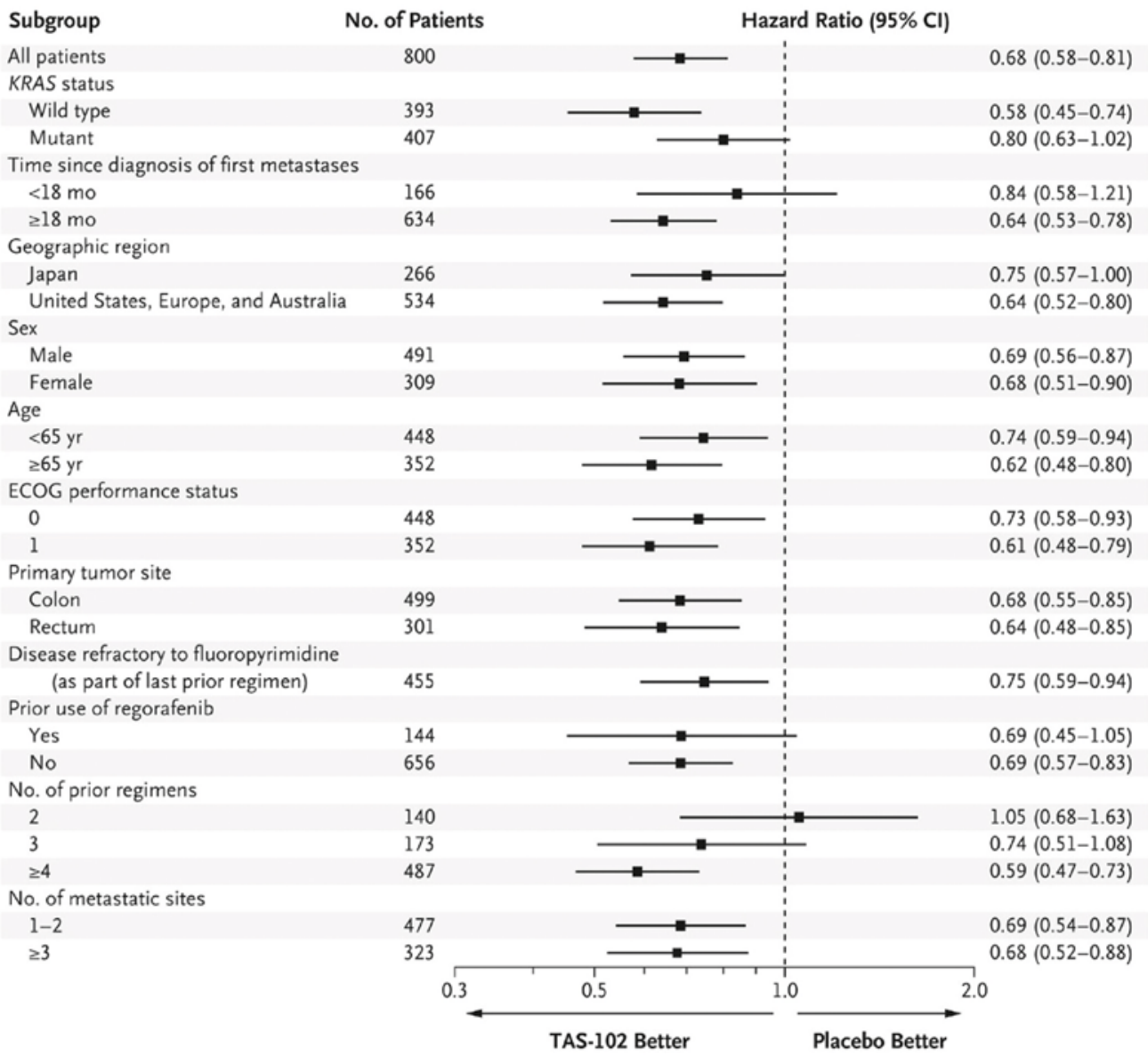

Fig. 2. OS of refractory mCRC patients. Patients treated with FTD/TPI showed a clear OS advantage compared to the placebo group. All of the patients showed a benefit from the treatment apart from those who had only 2 prior regimens. Adapted from Mayer et al. [12]. 
Fig. 3. Toxicity comparison between FTD/ TPI and regorafenib. Adapted from Tournigand [38].

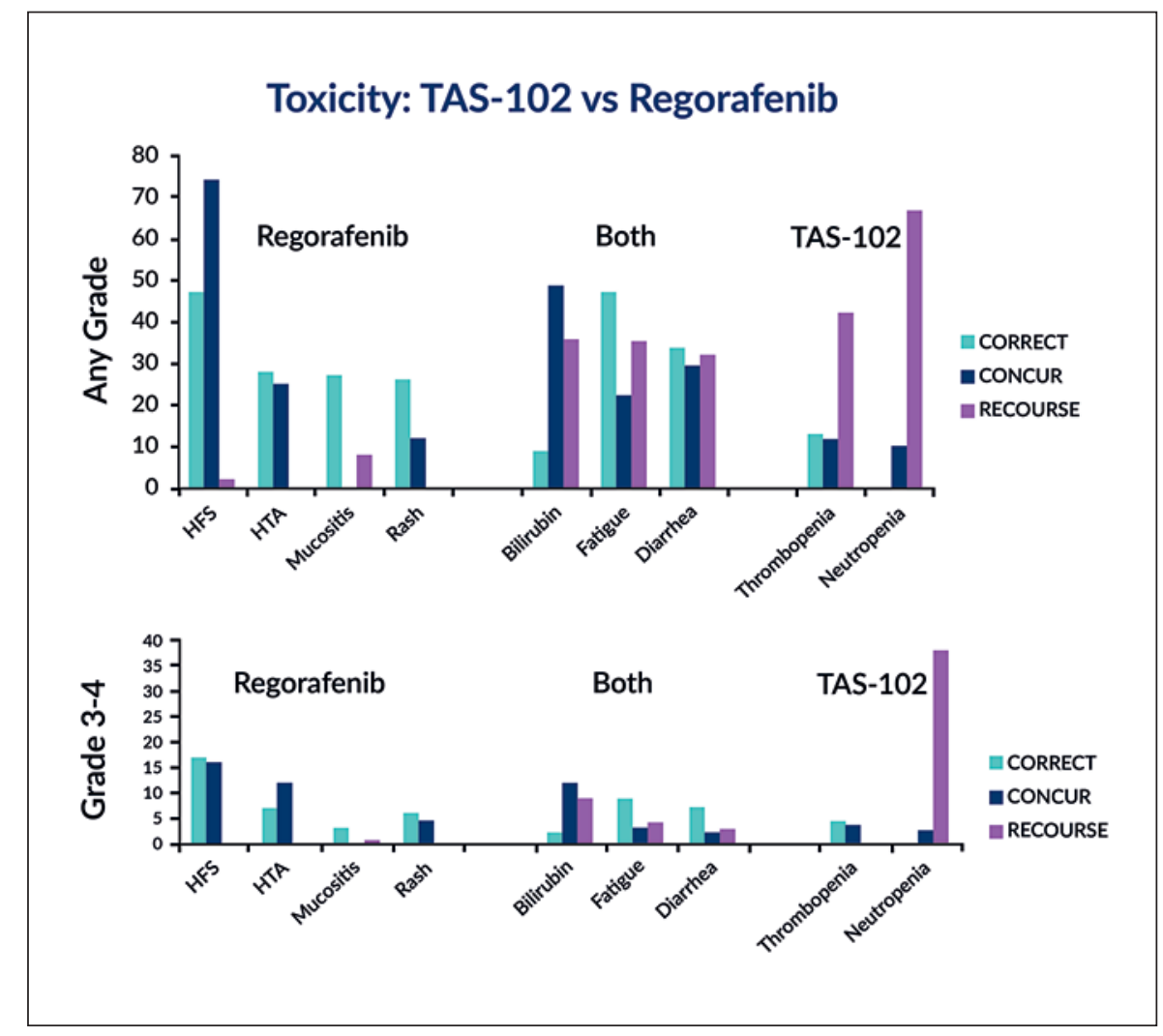

sponse rates (i.e., 21\%) and disease control rates (i.e., $54 \%$ ) were reported in the CRICKET study, but the median PFS was relatively short (i.e., 1.9 months in RASmutated patients and 3.9 months in KRAS wild-type patients) [25].

Rechallenge with oxaliplatin in $\mathrm{mCRC}$ can be an option in the refractory setting that can achieve tumor control, PFS, and OS benefits $[28,29]$. In this setting, however, patients should be monitored carefully for neurotoxicity, hypersensitivity, and allergic reactions [28]. Rechallenge with an oxaliplatin-based chemotherapy can therefore represent an option for the treatment of refractory $\mathrm{mCRC}$ and it is therefore listed in the joint ESMO [3], German S3 [4] and NCCN [5] guidelines. Rechallenge with a chemotherapy combination such as FOLFOX or FOLFIRI, however, has not been studied prospectively [22].

Overall, the evidence for the success of a rechallenge strategy with cetuximab or bevacizumab with or without chemotherapy such as oxaliplatin and irinotecan is limited. The results of such studies must be interpreted with caution due to the small sample sizes and a lack of appropriate controls [22]. Further prospective clinical trials are warranted before this option can routinely be considered in clinical practice. Factors that support the rechallenge strategy in $\mathrm{mCRC}$ patients are a long duration of response to a first-line anti-EGFR therapy, a good general performance status, the duration of the treatment response in the second line and an interval of at least 6 months between the first anti-EGFR therapy and the anti-EGFR rechallenge. However, molecular retesting is recommended for anti-EGFR re-challenge therapy. In addition, a positive effect on PFS was observed only in KRAS wild-type patients [25].

To date, there has been no direct comparison between rechallenge with a chemotherapy doublet and FTD/TPI or regorafenib. However, recent data suggest that the timely use of FTD/TPI or regorafenib in early phases of refractory $\mathrm{mCRC}$ is superior to renewed exposure to a chemotherapy doublet or anti-EGFR therapy with regard to the time to treatment failure and OS duration [22]. Furthermore, FTD/TPI and regorafenib were evaluated in large-scale phase 2 and 3 studies in mCRC after a second progression. Thus, these treatment options are now the preferred choice after a second progression and should be given prior to rechallenge therapy [22]. Moreover, a randomized phase 2 study compared cetuximab with regorafenib after prior treatment with fluoropyrimidines, irinotecan, and oxaliplatin [26]. This sequential treatment regimen resulted in a markedly longer OS compared to that of the reverse sequence (17.4 vs. 11.6 months, $\mathrm{HR}=0.61$ ) [26]. Apart from the danger of deterioration of the performance status over time, these results indicate that regorafenib and, by analogy, FTD/TPI should be used prior to rechallenge therapy with cetuximab. 


\section{The Safety and Quality-of-Life Profiles of FTD/TPI and Regorafenib Impact Treatment Decisions}

Currently, there are only limited data available on the optimal therapy sequence involving FTD/TPI and regorafenib. The key question is whether FTD/TPI or regorafenib should be administered first, and this was investigated indirectly in PRECONNECT [10, 30]. A post hoc subgroup analysis showed that the sequence of treatment with FTD/TPI and regorafenib did not affect the efficacy of the following treatment regimen [30]. In addition, there is no evidence to suggest that either of the 2 treatment options leads to better efficacy. Recent data from the RETAS study confirms that both agents can be administered in sequence [31]. Without a direct head-tohead comparison, the sequence of administration remains a clinical decision.

The authors of the PRECONNECT study concluded that the safety and quality-of-life profiles are important aspects to be considered when treating mCRC patients after a second progression. It is important to note that FTD/TPI has presented a favorable toxicity profile in comparison to regorafenib [30]. This also enables patients to receive further treatment lines in future.

With FTD/TPI, toxicities in the first 1-2 months are mostly hematological. For instance, neutropenia, anemia, and thrombocytopenia can occur quickly. In a study by Kasi et al. [32], it was observed that neutropenia induced by FTD/TPI treatment was associated with a better prognosis in patients with refractory mCRC. Thus, the extent of neutropenia in such patients can be instrumental in dose adjustments as well as in predicting treatment outcomes [32]. Toxicities associated with regorafenib, on the other hand, can be more severe (Fig. 3). Regorafenib is mostly associated with hand-and-foot syndrome, fatigue (which also might be observed after treatment with FTD/TPI, but in a milder form), liver toxicity, and hypertension. As these complaints usually occur in the first 1-2 weeks, patients on regorafenib need to visit the clinic once a week during the first month. Patients exhibiting pronounced fatigue at this stage should be monitored regularly [33].

Another factor affecting treatment decisions after a second progression is the impact of treatment on the quality of life of the patient. The PRECONNECT study shows that more than $20 \%$ of patients receiving FTD/TPI had improved EORTC Quality-Of-Life Core Questionnaire Core-30 (QCQ-C30) scores, and more than half of the patients $(58.5 \%)$ showed no deterioration in their quality of life [34]. Furthermore, the ECOG performance status was maintained after treatment with FTD/TPI compared to placebo $[2,10-12,30]$. With regorafenib, clinical studies have demonstrated that the quality of life did not deteriorate in patients treated with regorafenib compared to placebo $[22,33]$.

\section{Various Aspects to be Considered and Individualized Concepts}

As the treatment options for $\mathrm{mCRC}$ patients are evolving rapidly, several guidelines [3-5] recommend discussing the available treatment approaches within a multidisciplinary tumor board (MDT) consisting of experts in their field of treatment. This could also include individualized concepts. For oligometastatic liver disease, for instance, ESMO guidelines list various ablative modalities like stereotactic ablative body radiotherapy and radiofrequency ablation as well as hyperthermic intraperitoneal chemotherapy for peritoneal disease, and nodal dissection. Surgical procedures have also been expanded beyond the resection of maximally 3 liver metastases by novel methods like 2 -step associating liver partition and portal vein ligation for staged hepatectomy (ALPPS) for highly selected cases after discussion in an MDT. These scenarios highlight the complexity of an individualized concept using surgical or interventional steps in addition to the systemic options [3].

Tumor heterogeneity and clonal evolution add to the complexity of treating refractory mCRC. Therefore, rebiopsy of the primary or metastases should be considered $[25,35]$. However, this will rather be a topic of future clinical trials and not part of clinical routine as the therapeutic consequences are still unclear.

After anti-EGFR regimens have been exhausted in RAS wild-type mCRC, extended molecular testing should be taken into account at the latest in the refractory setting. Currently several commercial platforms are available for comprehensive tumor profiling, which detects various subtypes within a certain tumor entity. For mCRC the available molecularly driven therapies include BRAF- and Her-2-directed therapies as well as immune checkpoint inhibition for DNA-mismatch-repair-deficient tumors as well rare mutations (e.g., POLE mutations). This extended molecular testing might also uncover very rare but highly targetable mutations or alterations like NTRK fusions which allow treatment with highly selective drugs like entrectinib or larotrectinib [36]. Within ongoing trials considering early use of targeted treatments like BRAF-mutated $\mathrm{mCRC}$, extended molecular testing will be more focused on the primary diagnosis of mCRC in the future. In summary, treatment of mCRC will be become more complex in the future and, besides an MDT, treatment possibilities including expanded molecular profile testing should be discussed in a molecular tumor board.

\section{Future Perspectives}

A variety of treatment options depicted in the different guidelines are currently available to physicians treating patients with mCRC. While the development of these 
treatment options has improved the overall clinical outcome for such patients, the lack of a definitive and wellestablished treatment recommendation represents a challenge for physicians. Especially for Swiss oncologists this is a relevant factor, as there are no Swiss-specific guidelines for the management or treatment of mCRC in Swiss patients that also includes the availability of these drugs in the country as well regulatory affairs. This might be counterproductive for prolongation of OS or for improvement of general clinical outcomes for patients. The relevance of creating Switzerland-based recommendations regarding treatment sequences in relation to clinical, tumor biologi$\mathrm{cal}$, and regulation-based factors is of high interest.

Furthermore, an alternative approach would be to combine FTD/TPI with rechallenge therapy as a treatment option after a second progression. The APOLLON study evaluated the combination of FTD/TPI with an anti-EGFR antibody and this led to considerable improvement in the PFS (5.8 months) and response rate (37\%) with an acceptable safety profile [37]. However, this study had a limited data set as it included only patients without prior anti-EGFR treatment. Although the result appears promising, further analysis will be needed before a conclusion can be drawn on this combinatorial approach [37].

\section{Conclusions}

Treatment of mCRC in refractory settings remains a challenging field, with several options such as FTD/TPI, regorafenib, and rechallenges equally recommended in the updated guidelines $[4,5,22]$. There is currently no evidence to suggest a better efficacy of either treatment. However, tolerability and the quality of life of patients are also important factors to consider when choosing a treatment option after a second progression. Toxicity and impact on quality of life assessments for either regorafenib or FTD/TPI treatment options have shown that FTD/TPI has a favorable safety profile. Neither of the 2 treatments has any impact on the quality of life of the patient.

The strategy of giving FTD/TPI or regorafenib prior to rechallenge therapy allows for as many treatment lines as possible to achieve the maximal prolongation of OS in the patients [3, 4]. Based on the conducted studies FTD/TPI or regorafenib may be chosen earlier, although most of the study patients were treated after failure of at least 2 treatment lines. Rechallenge therapy, on the other hand, may be given in highly selected cases, i.e., in RAS wildtype patients who have shown a sustained benefit of therapy in an earlier line with disease control.

However, there is still a need for innovative treatments to supplement the currently available options. Moreover, new biomarkers are needed to facilitate the development of more tailored treatments to match individual patient needs. Such biomarkers will also play an important predictive role to better guide physicians in choosing the most effective treatment regimen. Lastly, study models can potentially determine the optimal treatment sequence, as large randomized studies will hardly be feasible for determining the most effective sequence of treatments.

In conclusion, FTD/TPI and regorafenib provide similar advantages in the treatment of refractory mCRC.

\section{Acknowledgment}

We thank our collaborators for their comments and inputs for preparation of the final version of this paper. These persons contributed equally: Daniel Helbling, Rahel Odermatt, Thomas Winder, and Philippe von Burg.

\section{Statement of Ethics}

Ethical approval was not necessary as this is a review.

\section{Disclosure Statement}

A.S. has received consulting honoraria from Amgen, Bayer, BMS, Eisai, IPSEN, Lilly, Merck, MSD, Novartis, Pfizer, Roche, Sanofi, and Servier and travel grants from Amgen, BMS, IPSEN, Roche, and Servier.

S.D. has received consulting honoraria from Amgen, Bayer, BMS, IPSEN, Lilly, Merck, BMS, Novartis, Pfizer, Roche, Sanofi, and Servier, and travel grants from Amgen, BMS, IPSEN, Roche and Servier.

A.M. has provided a consulting/advisory role for Astellas, Janssen, MSD, Roche, Celgene, Novartis, Vifor, Sanofi, Amgen, and Merck, has received research funding from Bayer (Personal) and MERCK (Inst), has intellectual property interests relating to Merck (not related to this report), has been paid to provide expert testimony for Sanofi and has reported travel/accommodation/other expenses paid for by Amgen, Astellas, Janssen, Servier, Sanofi, Roche, and Boehringer Ingelheim.

A.D.W. has received travel support from Ipsen, Abbvie, and Sanofi, and honoraria or consultation fees from Bayer, BMS, Merck, Servier, and Lilly. She is coordinating investigator 55 of the EORTC 1203 trial, which is supported by an educational grant from Roche to EORTC.

M.B. has nothing to disclose.

\section{Funding Sources}

This research did not receive any specific grant from funding agencies in the public, commercial, or not-for-profit sector. $\mathrm{H}+\mathrm{O}$ Communication AG supported to generate the figures of this publication.

\section{Author Contributions}

All of the authors designed this study, analyzed the data, wrote this paper, and gave final approval of the submitted version. 


\section{References}

1 PDQ Screening and Prevention Editorial Board. Colorectal cancer screening (PDQ): health professional version - PDQ cancer information summaries. Bethesda: National Cancer Institute (US); 2002.

2 Bordoni A, Lorez M, Bouchardy C, Konzelmann I, Clough-Gorr K; NICER Working Group. Trends in colorectal cancer survival in Switzerland. Zurich: Nationales Institut für Krebsepidemiologie und -registrierung. 2012.

3 Van Cutsem E, Cervantes A, Adam R, Sobrero A, Van Krieken JH, Aderka D, et al. ESMO consensus guidelines for the management of patients with metastatic colorectal cancer. Ann Oncol. 2016 Aug;27(8):1386-422.

4 Leitlinienprogramm Onkologie. S3-Leitlinie Kolorektales Karzinom. Berlin: DKG; 2019.

5 NCCN Guidelines ${ }^{\circledR}$. NCCN clinical practice guidelines in oncology: colon cancer - version 1.2019. Plymouth Meeting: National Comprehensive Cancer Network; 2019.

6 Dekker E, Tanis PJ, Vleugels JLA, Kasi PM, Wallace MB. Colorectal cancer. Lancet. 2019 Oct 19;394(10207):1467-80.

7 Kirstein MM, Lange A, Prenzler A, Manns MP, Kubicka S, Vogel A. Targeted therapies in metastatic colorectal cancer: a systematic review and assessment of currently available data. Oncologist. 2014 Nov;19(11):1156-68.

8 Lenz HJ, Stintzing S, Loupakis F. TAS-102, a novel antitumor agent: a review of the mechanism of action. Cancer Treat Rev. 2015 Nov; 41(9):777-83.

9 Characteristics of Lonsurf ${ }^{\circledR}$ : summary of product characteristics of Lonsurf $^{\circledR}$ [Internet]. 2017. Available from: http://www.swissmedicinfo.ch.

10 Falcone A, André T, Edeline J, François E, Taieb J, Phelip J, et al. Safety and efficacy of trifluridine/tipiracil in previously treated metastatic colorectal cancer (mCRC): preliminary results from the phase IIIb, international, open-label, early-access PRECONNECT study. Ann Oncol. 2018;29:v104-5.

11 Van Cutsem E, Mayer RJ, Laurent S, Winkler R, Grávalos C, Benavides M, et al.; RECOURSE Study Group. The subgroups of the phase III RECOURSE trial of trifluridine/tipiracil (TAS-102) versus placebo with best supportive care in patients with metastatic colorectal cancer. Eur J Cancer. 2018 Feb;90:63-72.

12 Mayer RJ, Van Cutsem E, Falcone A, Yoshino T, Garcia-Carbonero R, Mizunuma N, et al. RECOURSE Study Group. Randomized trial of TAS-102 for refractory metastatic colorectal cancer. N Engl J Med. 2015 May;372(20): 1909-19.

13 Ohtsu A, Yoshino T, Falcone A, Garcia-Carbonero R, Argiles G, Sobrero AF, et al. Onset of neutropenia as an indicator of treatment response in the phase 3 RECOURSE trial of trifluridine/tipiracil (TAS-102) versus placebo in patients with metastatic colorectal cancer. J Clin Oncol. 2017;35:775.

14 Pfeiffer P, Yilmaz M, Möller S, Maltha L, Krogh M, Zitnjak D, et al. Randomized study evaluating trifluridine/tipiracil (TAS-102) versus + trifluridine/tipiracil + bevacizumab as last-line therapy in patients with chemorefractory unresectable metastatic colorectal cancer (mCRC). J Clin Oncol. 2019;37:637.
15 Kuboki Y, Nishina T, Shinozaki E, Yamazaki K, Shitara K, Okamoto W, et al. TAS-102 plus bevacizumab for patients with metastatic colorectal cancer refractory to standard therapies (CTASK FORCE): an investigator-initiated, open-label, single-arm, multicentre, phase $1 / 2$ study. Lancet Oncol. 2017 Sep;18(9):1172-81.

16 Salah-Eddin AB. Ramucirumab in combination with TAS102 vs. TAS102 alone in chemotherapy refractory metastatic colorectal cancer patients (RAMTAS). ClinicalTrials.gov registration number: NCT03520946. Forthcoming.

17 Skuja E, Gerina-Berzina A, Hegmane A, Zvirbule Z, Vecvagare E, Purkalne G. Duration of previous treatment as a prognostic factor in metastatic colorectal cancer treated with trifluridine/tipiracil. Mol Clin Oncol. 2018 May; 8(5):699-702.

18 Cremolini C, Rossini D, Martinelli E, Pietrantonio F, Lonardi S, Noventa S, et al. Trifluridine/ Tipiracil (TAS-102) in Refractory Metastatic Colorectal Cancer: A Multicenter Register in the Frame of the Italian Compassionate Use Program. Oncologist. 2018 Oct;23(10):1178-87.

19 Kwakman JJ, Vink G, Vestjens JH, Beerepoot LV, de Groot JW, Jansen RL, et al. Feasibility and effectiveness of trifluridine/tipiracil in metastatic colorectal cancer: real-life data from The Netherlands. Int J Clin Oncol. 2018 Jun;23(3):482-9.

20 Kasper S, Kisro J, Fuchs M, Müller C, SchulzAbelius A, Karthaus M, et al. Safety profile of trifluridine/tipiracil monotherapy in clinical practice: results of the German compassionate-use program for patients with metastatic colorectal cancer. BMC Cancer. 2018 Nov; 18(1):1124.

21 Chiang CL, Choi HC, Lam KO, Chan BY, Lee SF, Yeung SY, et al. Real-world treatment patterns and outcomes in refractory metastatic colorectal cancer. Asia Pac J Clin Oncol. 2019 Mar;15(S2 Suppl 2):5-13.

22 Arnold D, Prager GW, Quintela A, Stein A, Moreno Vera S, Mounedji N, et al. Beyond second-line therapy in patients with metastatic colorectal cancer: a systematic review. Ann Oncol. 2018 Apr;29(4):835-56.

23 Santini D, Vincenzi B, Addeo R, Garufi C, Masi G, Scartozzi M, et al. Cetuximab rechallenge in metastatic colorectal cancer patients: how to come away from acquired resistance? Ann Oncol. 2012 Sep;23(9):2313-8.

24 Tonini G, Imperatori M, Vincenzi B, Frezza AM, Santini D. Rechallenge therapy and treatment holiday: different strategies in management of metastatic colorectal cancer. J Exp Clin Cancer Res. 2013 Nov;32(1):92.

25 Rossini D, Cremolini C, Conca E, Del Re M, Busico A, Pietrantonio F, et al. Liquid biopsy to predict benefit from rechallenge with cetuximab (cet) + irinotecan (iri) in RAS/BRAF wild-type metastatic colorectal cancer patients (pts) with acquired resistance to first-line cet+iri: final results and translational analyses of the CRICKET study by GONO. J Clin Oncol. 2018;36:12007.

26 Shitara K, Yamanaka T, Denda T, Tsuji Y, Shinozaki K, Komatsu Y, et al. REVERCE: randomized phase II study of regorafenib followed by cetuximab versus the reverse sequence for metastatic colorectal cancer patients previously treated with fluoropyrimidine, oxaliplatin, and irinotecan. J Clin Oncol. 2018;36:557.

27 Wirapati P, Pomella V, Vandenbosch B, Kerr P, Maiello E, Jeffery GM, et al. Velour trial biomarkers update: impact of RAS, BRAF, and sidedness on aflibercept activity. J Clin Oncol. 2017;35:3538.

28 Kim JJ, Kang J, Hong YS, Kim KP, Kim SY, Kim TW, et al. Oxaliplatin rechallenge in metastatic colorectal cancer patients after prior oxaliplatin treatment. Med Oncol. 2018 Apr;35(5):65.

29 Yang Q, Huang Y, Jiang Z, Wang H, Li W, Zhang B, et al. Rechallenge of oxaliplatin-containing regimens in the third- or later-line therapy for patients with heavily treated metastatic colorectal cancer. Onco Targets Ther. 2018 May;11:2467-73.

30 Taieb J, Falcone A, Lonardi S, Price T, Bachet J, Wyrwicz L, et al. Safety and efficacy of trifluridine/tipiracil (FTD/TPI) in metastatic colorectal cancer (mCRC) patients according to previous treatment with regorafenib in the international phase IIIb PRECONNECT study. Ann Oncol. 2018;29:464P.

31 Masuishi T, Taniguchi H, Hamauchi S, Komori A, Kito Y, Narita Y, et al. Regorafenib Versus Trifluridine/Tipiracil for Refractory Metastatic Colorectal Cancer: A Retrospective Comparison. Clin Colorectal Cancer. 2017 Jun;16(2):e15-22.

32 Kasi PM, Kotani D, Cecchini M, Shitara K, Ohtsu A, Ramanathan RK, et al. Chemotherapy induced neutropenia at 1-month mark is a predictor of overall survival in patients receiving TAS102 for refractory metastatic colorectal cancer: a cohort study. BMC Cancer. 2016 Jul;16(1):467.

33 Grothey A, Van Cutsem E, Sobrero A, Siena S, Falcone A, Ychou M, et al.; CORRECT Study Group. Regorafenib monotherapy for previously treated metastatic colorectal cancer (CORRECT): an international, multicentre, randomised, placebo-controlled, phase 3 trial. Lancet. 2013 Jan;381(9863):303-12.

34 Taieb J, Price TJ, Ciardiello F, Peeters M, Wyrwicz L, Bachet J-B, et al. Health-related quality of life in the early-access phase IIIb study of trifluridine/tipiracil in pretreated metastatic colorectal cancer (mCRC): results from PRECONNECT study. J Clin Oncol. 2019;37:638.

35 Pietrantonio F, Vernieri C, Siravegna G, Mennitto A, Berenato R, Perrone F, et al. Heterogeneity of acquired resistance to anti-EGFR monoclonal antibodies in patients with metastatic colorectal cancer. Clin Cancer Res. 2017;23(10):2414-22.

36 Taieb J, Jung A, Sartore-Bianchi A, Peeters M, Seligmann J, Zaanan A, et al. The Evolving Biomarker Landscape for Treatment Selection in Metastatic Colorectal Cancer. Drugs. 2019 Sep;79(13):1375-94.

37 Kuboki Y, Yoshino T, Kato T, Kagawa Y, Gamoh M, Yasui H, et al. APOLLON: a phase I/II study of panitumumab combined with TAS-102 in patients (pts) with RAS wild-type (wt) metastatic colorectal cancer (mCRC). J Clin Oncol. 2018;36(15_suppl):3523-.

38 Tournigand C. ESMO Abstracts 5000 and LBA13 slide 16. 2014. Available from: https:// cslide.ctimeetingtech.com/library/esmo/ browse/search/zfC-9f8U03Ad. 